Revue internationale P.M.E.

Économie et gestion de la petite et moyenne entreprise

Revure

internationale

PME

\title{
Le coût du capital entrepreneurial
}

\section{Cécile Carpentier et Jean-Marc Suret}

Volume 24, numéro 3-4, 2011

URI : https://id.erudit.org/iderudit/1013664ar

DOI : https://doi.org/10.7202/1013664ar

Aller au sommaire du numéro

Éditeur(s)

Presses de l’Université du Québec

ISSN

0776-5436 (imprimé)

1918-9699 (numérique)

Découvrir la revue

Citer cet article

Carpentier, C. \& Suret, J.-M. (2011). Le coût du capital entrepreneurial. Revue internationale P.M.E., 24(3-4), 103-136. https://doi.org/10.7202/1013664ar

\section{Résumé de l'article}

Le coût du financement par les fonds propres (coût du capital) est un élément essentiel de l'évaluation de l'entreprise, mais aussi des opérations de financement et de l'analyse des projets. Toutefois, estimer le coût du capital entrepreneurial est un exercice extrêmement difficile. Les modèles classiques de la finance, adaptés aux grandes entreprises, ne peuvent pas s'appliquer dans ce cadre particulier. Les données de marché, essentielles aux estimations, sont inexistantes. Nous présentons, illustrons et critiquons les principales méthodes proposées en la matière. Nous proposons par ailleurs la seule estimation récente des primes de taille applicable à des entreprises de très faible capitalisation. Ni les pratiques ni les modèles théoriques ne fournissent actuellement de solution satisfaisante au problème de l'estimation du coût du capital entrepreneurial. Nous suggérons donc plusieurs avenues de recherche visant à mieux comprendre et estimer ce coût.
Ce document est protégé par la loi sur le droit d'auteur. L'utilisation des services d’Érudit (y compris la reproduction) est assujettie à sa politique d'utilisation que vous pouvez consulter en ligne.

https://apropos.erudit.org/fr/usagers/politique-dutilisation/ 


\title{
Le coût du capital entrepreneurial
}

\author{
Cécile CARPENTIER \\ Jean-Marc SURET \\ Université Laval, Fellow CIRANO et European Center \\ for Corporate Control Studies
}

\section{MOTS CLÉS}

Coût des fonds propres - Entrepreneuriat - PME - Estimation Prime de taille

\begin{abstract}
LES AUTEURS
CÉCILE CARPENTIER est professeure à I'Université Laval et Fellow CIRANO (Canada). Ses recherches portent sur le financement des petites entreprises et sur les politiques publiques. Elle a publié des rapports et des articles relatifs aux premiers appels publics à l'épargne, aux sociétés de capital de démarrage, aux fusions inversées, aux placements périodiques de titres de participation au Canada et à la Bourse de croissance TSX. Elle a publié des articles dans plusieurs revues scientifiques telles que Journal of Business Venturing, International Small Business Journal, Small Business Economics, Venture Capital, Canadian Tax Journal et Canadian Public Policy. Adresse: Université Laval, Faculté des sciences de l'administration, École de comptabilité, Pavillon Palasis-Prince, bureau 6244, Québec (Québec), Canada, G1V 0A6. Courriel: <cecile.carpentier@fsa.ulaval.ca>.

JEAN-MARC SURET est professeur à l'Université Laval et Fellow CIRANO (Canada). Ses thèmes de recherche de prédilection portent sur la finance d'entreprise, les politiques publiques et la réglementation. II s'intéresse surtout à l'équilibre entre la protection des investisseurs et le financement des petites entreprises. II a publié de nombreux articles dans des revues scientifiques telles que Journal of Business Venturing, Contemporary Accounting Research, Empirical Legal Studies, Small Business Economics, Journal of International Business Study, Venture Capital, Financial Review, Canadian Tax Journal, Canadian Public Policy et Journal of Private Equity. Adresse: Université Laval, Faculté des sciences de l'administration, École de comptabilité, Pavillon Palasis-Prince, bureau 6226, Québec (Québec), Canada, G1V 0A6. Courriel: <jean-marc.suret@fsa.ulaval.ca>.
\end{abstract}

\section{RÉSUMÉ}

Le coût du financement par les fonds propres (coût du capital) est un élément essentiel de l'évaluation de l'entreprise, mais aussi des opérations de financement et de l'analyse des projets. Toutefois, estimer le coût du capital entrepreneurial est un exercice extrêmement difficile. Les modèles classiques de la finance, adaptés aux grandes entreprises, ne peuvent pas s'appliquer dans ce cadre particulier. 
Les données de marché, essentielles aux estimations, sont inexistantes. Nous présentons, illustrons et critiquons les principales méthodes proposées en la matière. Nous proposons par ailleurs la seule estimation récente des primes de taille applicable à des entreprises de très faible capitalisation. Ni les pratiques ni les modèles théoriques ne fournissent actuellement de solution satisfaisante au problème de l'estimation du coût du capital entrepreneurial. Nous suggérons donc plusieurs avenues de recherche visant à mieux comprendre et estimer ce coût.

\begin{abstract}
The equity cost of capital is crucial to business valuation, financing operations and project analyses, yet assessing this cost is very difficult. Classic financial models fit large firms well, but are not applicable to this context. Market data, which are pivotal to the estimates, are lacking. We present, illustrate and discuss the main methods proposed to assess the entrepreneurial equity cost. We also propose the only recent size premium estimates adapted to firms with very small market capitalization. Currently, neither practices nor the theoretical models solve the entrepreneurial equity cost measurement problem adequately. We therefore suggest research avenues to better understand and estimate this cost.
\end{abstract}

\title{
RESUMEN
}

El costo del capital propio es una parte esencial de la evaluación de la empresa, pero también de las operaciones de financiación y el análisis de los proyectos. Sin embargo, evaluar el costo del capital empresarial es muy complicado. Los modelos clásicos en finanzas, adaptados para las grandes empresas, no pueden aplicarse a este contexto particular. Los datos de mercado, esenciales para las estimaciones, no existen. Presentamos, ilustramos y criticamos los principales métodos propuestos en este campo. Proponemos además la única estimación reciente de las primas de tamaño aplicable a empresas de capitalización muy baja. Las prácticas y los modelos teóricos actuales no brindan soluciones convenientes al problema de la estimación del costo del capital empresarial. Por lo tanto, recomendamos varias opciones de exploración orientadas a comprender y estimar mejor dicho costo.

\section{ZUSAMMENFASSUNG}

Die Eigenkapitalkosten sind ein wesentliches Element zur Bewertung von Unternehmen und von Projekten. Die Bewertung der Kapitalkosten bei Unternehmensgründungen hingegen ist als extrem schwierig zu bezeichnen. Klassische Finanzierungsmodelle, gedacht für Grossunternehmen, eigenen sich nicht für den speziellen unternehmerischen Kontext. Marktdaten, essentiell zur Bewertung, sind schlicht nicht verfügbar. Wir präsentieren, illustrieren und kritisieren die bedeutendsten Bewertungsansätze. Weder die gängigen Praktiken noch theoretische Modelle bieten eine befriedigende Lösung für die Bewertung von unternehmerischem Kapital. Wir schlagen daher verschiedene Forschungsperspektiven vor, um die damit verbundenen Probleme besser zu verstehen. 


\section{Introduction}

Le coût du capital entrepreneurial est le taux de rendement exigé par les investisseurs pour placer leurs fonds dans une petite entreprise à forte croissance, une entrepreneurial venture. C'est le taux auquel l'entrepreneur potentiel peut se référer lorsqu'il se demande si son projet mérite d'être développé. Le coût du capital est le rendement qu'il serait équitable d'offrir aux investisseurs relationnels, parents et amis. Il est au centre des négociations avec les investisseurs informels, les anges investisseurs ${ }^{1}$. Le promoteur doit en effet proposer un montage offrant aux investisseurs un rendement satisfaisant. Le coût du capital est également l'élément clé de l'évaluation de l'entreprise. À ce titre, il intervient dans les jugements relatifs aux litiges portant sur la valeur des actions de sociétés fermées. Lorsque l'évaluation intervient dans un contexte de financement, elle détermine la proportion des actions à céder en contrepartie de la mise de fonds des investisseurs. Par ailleurs, connaître l'ordre de grandeur du coût du capital entrepreneurial permet de comprendre pourquoi le financement externe par les capitaux propres restera une option coûteuse réservée à une minorité d'entrepreneurs.

En dépit de l'importance de ce sujet, très peu de travaux concernant le coût du capital entrepreneurial ${ }^{2}$. Ce thème est d'ailleurs absent des revues de la littérature en finance entrepreneuriale (Denis, 2004; Redis et Paré, 2009). Les rares travaux publiés s'intéressent essentiellement au cas des sociétés de capital de risque, qui ne financent annuellement que quelques centaines d'entreprises, aux caractéristiques très particulières (Angel Capital Education Fundation, 2010). Hormis le volume de Smith, Smith et Bliss (2011), les manuels de finance entrepreneuriale sont également fort discrets sur ce sujet, qui n'est parfois même pas abordé. L'estimation de ce coût est pourtant particulièrement délicate puisqu'on ne dispose ni de données de marché sur lesquels baser les calculs ni de modèles applicables à leur situation.

1. On désigne ainsi des investisseurs externes n'ayant ni lien de parenté ni relation amicale avec l'entrepreneur. Ce sont généralement des individus fortunés ayant une expérience des affaires, qui participent à la gestion des entreprises dans lesquelles ils investissent.

2. Parmi les 512 documents apparaissant en recherchant le terme «cost of equity» sur SSRN, quatre seulement subsistent lorsqu'on ajoute les mots clés «SME», «entrepreneurial» ou encore «start-up». Une analyse des textes référencés sous «entrepreneurial finance» dans la même base révèle quatre articles et une note pédagogique traitant du coût du capital. 
Dans cet article, nous passons en revue les possibilités offertes pour estimer le coût du capital entrepreneurial et en illustrons les bases conceptuelles, les démarches et les limites. Nous tentons également de montrer les pistes de recherche à explorer. Nos contributions sont les suivantes: nous présentons la première vue d'ensemble critique des diverses méthodes évoquées par les chercheurs ou utilisées par les praticiens pour estimer le coût du capital entrepreneurial, nous établissons les liens entre ces diverses approches, nous récapitulons les principaux résultats des analyses du taux de rendement effectif de ce capital et nous proposons des estimations originales de la prime de taille associée aux titres de très petite capitalisation, utilisables pour estimer le coût du capital des PME.

Dans la première section, nous définissons le capital entrepreneurial et relevons les limites des approches traditionnelles d'estimation du coût du capital. Nous présentons dans une deuxième section l'origine des difficultés d'estimation du coût du capital entrepreneurial. Nous abordons ensuite successivement, et par ordre de complexité, quatre approches utilisables pour tenter d'estimer ce coût. La première consiste à empiler des primes arbitraires jusqu'à obtenir un taux jugé raisonnable (section 3). La deuxième demande l'estimation empirique des primes associées aux divers facteurs de risque (section 4). Les approches en risque total tentent de mieux quantifier le risque spécifique (section 5), qui peut également être modélisé en utilisant les probabilités d'échec des entreprises (section 6). Nous proposons quelques avenues de recherche en conclusion.

\section{Coût du capital entrepreneurial: limites des approches traditionnelles}

\subsection{Capital entrepreneurial}

L'entrepreneuriat implique la découverte, l'évaluation et l'exploitation de possibilités pour introduire de nouveaux biens ou services, structures d'organisation ou marchés, en rassemblant des ressources qui n'avaient pas été, antérieurement, utilisées de cette façon (Shane, 2003, p. 4). Le financement entrepreneurial concerne l'obtention des ressources financières requises par ce processus de développement. Il est habituellement associé à l'obtention de capitaux propres, car les entreprises ne sont alors généralement ni rentables ni détentrices d'éléments d'actif tangibles pouvant garantir un emprunt (Denis, 2004, p. 304; Gompers et Lerner, 2010). Les phases de financement du démarrage, de la croissance ainsi que la sortie des investisseurs lors de l'émission initiale constituent les principales composantes du financement entrepreneurial (Smith et al., 2011; Yazdipour, 2011). 
Nous considérons que le financement entrepreneurial concerne l'obtention des capitaux nécessaires entre la création de la nouvelle entreprise et l'établissement sur un marché public classique, ou encore la cession de l'entreprise à une autre entité. Il concerne des entreprises en croissance; ces entreprises sont le plus souvent fermées. Les apporteurs de capitaux sont les amis, la famille, les anges investisseurs, les sociétés de capital de risque et d'autres entreprises (Denis, 2004). Toutefois, le capital obtenu par l'intermédiaire des marchés juniors est souvent considéré comme entrepreneurial (Locke et Gupta, 2008). Diverses études, incluant le texte de référence en matière de rentabilité du capital entrepreneurial (Moskowitz et Vissing-Jorgensen, 2002), désignent ainsi l'ensemble des fonds investis dans des PME. Dans ce texte, à la suite de la définition utilisée par la Commission européenne (2003) pour traduire entrepreneurial venture, le capital entrepreneurial désigne les capitaux propres requis par des PME en forte croissance, fermées ou inscrites sur une bourse réservée aux entreprises de croissance.

\subsection{Mesures classiques}

Le coût du capital est le taux de rendement requis par les agents qui investissent une partie de leur épargne en actions d'entreprises. C'est un coût de renonciation: le taux auquel les actionnaires renoncent pour investir dans l'entreprise; ce coût est fonction du risque de l'entreprise. En principe, ce coût est le même pour le propriétaire-dirigeant et pour l'investisseur externe, puisqu'ils font face au même risque. Toutefois, la forme de la rémunération du capital peut différer.

Pour évaluer ce coût, il faut une estimation du risque et un modèle reliant ce risque au taux requis par les actionnaires. L'outil de référence classique dans l'univers des grandes entreprises ouvertes ${ }^{3}$ est le Capital Asset Pricing Model (CAPM), qui établit une relation linéaire entre le taux de rendement requis sur un titre $i$ et son risque systématique noté $\beta_{i}$.

$\mathrm{E}\left(\mathrm{R}_{i}\right)=\mathrm{r}_{f}+\beta_{i}\left(\mathrm{E}\left(\mathrm{R}_{M}\right)-\mathrm{r}_{f}\right)$

3. La société ouverte est inscrite en bourse et ses titres sont échangés librement; elle est un émetteur assujetti. La société fermée, quant à elle, ne peut pas vendre d'actions au public. Elle ne peut placer ses titres qu'auprès de certains investisseurs qualifiés (accredited investors), dont les anges investisseurs et les sociétés de capital de risque. 
Ce modèle est présenté dans la plupart des manuels de finance. Il nécessite de mesurer successivement le taux sans risque, le bêta et la prime de risque. Le taux sans risque est, en théorie, celui des bons du Trésor, mais il est courant d'utiliser le taux des obligations à 10 ans du gouvernement (Damodaran, 2008).

Les bêtas des titres varient selon le secteur et, dans un même secteur, en fonction du risque d'opération et de l'endettement (dimension omise ici). Ils sont calculés grâce aux rendements boursiers passés ou obtenus directement auprès de divers services d'information financière. La prime par unité de risque est l'écart prévu entre le rendement du marché boursier et le taux sans risque. Elle varie et son estimation est difficile (Damodaran, 2010).

Le CAPM ne suffit toutefois pas à expliquer le rendement des titres. Le coût du capital serait lié au risque systématique, mais également à différents facteurs non prévus par la théorie, tels que la taille, la croissance, la liquidité ou l'investissement. Les petites entreprises cotées procurent, en moyenne au cours de longues périodes, un rendement supérieur à celui des grandes entreprises. On ajoute désormais fréquemment une prime de taille au coût du capital estimé par le CAPM.

$\mathrm{E}\left(\mathrm{R}_{i}\right)=\mathrm{r}_{f}+\beta_{i}\left(\mathrm{E}\left(\mathrm{R}_{M}\right)-\mathrm{r}_{f}\right)+$ prime de taille

Cette prime repose sur le calcul des rendements moyens des actions regroupées en fonction de leur taille. La plupart des estimations sont effectuées sur le marché américain, avec des données d'actions inscrites sur les principales bourses. Les titres sont regroupés en 10 ou 25 catégories de taille. La prime est nulle pour la catégorie centrale et de 4 à $5 \%$ pour les petites entreprises (Damodaran, 2010, p. 34).

\subsection{Effet de taille et capital entrepreneurial}

L'ajustement pour l'effet de taille n'apporte toutefois pas de réponse satisfaisante en matière de coût du capital entrepreneurial, pour plusieurs raisons. La taille n'est pas la seule différence entre l'entreprise qui démarre et la grande entreprise cotée. La première fait face aux risques du développement des produits et des marchés, de l'étroitesse des équipes de direction, du manque de liquidité, et aux phénomènes d'agence et d'asymétrie informationnelle bien différents de ceux de la grande entreprise. Les estimations des primes de taille applicables aux très petites sociétés sont inexistantes. L'entreprise qui démarre se situe dans un cadre réglementaire 
et institutionnel différent de celui de la grande entreprise. Les estimations de coût du capital de sociétés fermées reposant sur l'ajout d'une prime de taille ont été parfois acceptées, modifiées ou refusées par les tribunaux américains (Pratt, 2009, p. 329).

\section{Le cas particulier des petites entreprises}

Les estimations et méthodes appliquées aux sociétés cotées ne peuvent pas être utilisées dans le cas des PME pour trois raisons:1) certaines hypothèses importantes des modèles classiques ne sont pas respectées dans l'univers des PME;2) l'application des modèles classiques et les résultats des observations ne peuvent être conciliés; 3 ) les données requises pour estimer le coût du capital ne sont généralement pas disponibles.

\subsection{Les limites conceptuelles des méthodes classiques}

Le CAPM repose sur plusieurs hypothèses. Sa dérivation demande notamment que les investisseurs aient des anticipations homogènes quant à la distribution des probabilités de flux monétaires. Dans le cas contraire, il devient impossible d'agréger les fonctions de demande individuelles pour parvenir à une situation d'équilibre du marché (L'Her et Suret,1991). Cette hypothèse n'est pas parfaitement respectée dans le cas des grandes entreprises: si les anticipations étaient homogènes, il n'y aurait ni dispersion des prévisions des analystes ni transactions sur les marchés. Toutefois, pour les sociétés ouvertes, plusieurs mécanismes permettent de résoudre ces apparentes contradictions. De nombreux travaux montrent comment les informations véhiculées par les transactions, et donc par les prix des actions échangées sur les marchés, permettent des anticipations de plus en plus homogènes, sans toutefois tendre vers un consensus parfait (Hellwig, 1980). Ce mécanisme d'agrégation de l'information par les prix fonctionne tant que les investisseurs optimistes et pessimistes peuvent s'exprimer. Ce n'est pas le cas lorsque les ventes à découvert sont limitées ou impossibles (L'Her et Suret, 1995) ou lorsqu'il n'existe pas d'options de vente. Dans une telle situation, les investisseurs pessimistes quant à l'évolution future d'un titre ne peuvent pas profiter de la baisse de valeur qu'ils anticipent, et les prix ne reflètent plus l'ensemble des anticipations. Le cadre des anticipations hétérogènes sans possibilité de ventes à découvert développé par Miller (1977) est d'ailleurs fréquemment exploité pour expliquer les phénomènes entourant les émissions initiales d'actions (Dong, Michel et Pandes, 2011). Les sociétés fermées présentent donc une situation très particulière au regard de l'hypothèse des anticipations homogènes. Aucune agrégation de l'information par les prix n'est possible, 
puisque les actions ne sont pas échangées et que ni les ventes à découvert ni les options ne peuvent être employées. Les dirigeants et les investisseurs externes auront donc, le plus souvent, des anticipations très différentes qui ne convergeront pas. Le rendement ne peut alors plus être modélisé dans un cadre de CAPM.

La situation est semblable en matière de diversification. Si, dans le cadre du CAPM, on suppose que les investisseurs détiennent le portefeuille de marché, cette hypothèse semble ne pas être vérifiée dans les faits: $90 \%$ des investisseurs détiennent moins de cinq titres (Séjourné, 2006). Toutefois, les particuliers investissent maintenant fréquemment en actions de fonds communs de placement: $88 \%$ des investisseurs canadiens en détiennent, selon Deaves, Dine et Horton (2006). Même s'ils n'achètent que peu d'actions, les investisseurs s'approchent du portefeuille de marché grâce aux instruments de placement collectifs. Le marché n'a donc pas à rémunérer un risque lié à l'absence de diversification, puisque les instruments permettant cette diversification sont simples et peu onéreux. Cette solution est le plus souvent hors d'atteinte de l'entrepreneur, qui devra concentrer ses avoirs dans les capitaux propres de l'entreprise. Dans un tel cas, le coût du capital peut être déterminé par des risques non systématiques.

\subsection{Les effets du manque de liquidité}

Les modèles classiques supposent généralement que les actions peuvent être rapidement échangées à un faible coût, permettant les opérations d'arbitrage, l'atteinte de l'équilibre et la transmission de l'information. Ce n'est pas le cas dans l'univers entrepreneurial, et le manque de liquidité constitue un problème important. En acquérant des actions pour lesquelles il n'existe pas de marché organisé, l'investisseur accepte un risque supplémentaire, se traduisant par une prime augmentant le coût du capital. La prime pour le manque de liquidité induit un escompte sur la valeur des actions de l'entreprise. Plusieurs ensembles de travaux empiriques aux résultats contrastés ont traité de ce problème, et les analyser correctement dans le cadre d'un seul article est impossible. À notre connaissance, il n'existe aucun modèle ni résultat généralisable permettant d'ajuster le coût du capital entrepreneurial pour le manque de liquidité. Cette situation découle en partie du fait que la taille, la liquidité et le risque sont des caractéristiques liées dont il est difficile d'isoler les effets.

La liquidité influence les taux de rendement requis par les investisseurs des sociétés cotées (Acharya et Pedersen, 2005; Liu, 2006). Liu (2006) estime l'écart de rendement entre les titres inscrits en bourse à faible liquidité et ceux à forte liquidité à $3 \%$ par année environ après ajustement pour la taille et la croissance. L'effet de liquidité explique pourquoi les titres s'apprécient 
(se déprécient) lors de leur inclusion (exclusion) dans un indice boursier (Amihud, Mendelson et Pedersen, 2005). Les estimations de la prime pour le manque de liquidité dans le secteur du placement privé sont du même ordre que celles obtenues pour les actions inscrites en bourse (Franzoni, Nowak et Phalippou, 2011).

Comme il est très difficile de mesurer le rendement ou le coût du capital d'entreprises fermées, l'essentiel des travaux en ce domaine a porté sur l'escompte qui pénaliserait les sociétés fermées par rapport aux sociétés ouvertes dont les titres sont fréquemment échangés. Les estimations empiriques reposent sur plusieurs types de transactions: les ventes d'actions d'entreprises ouvertes ayant des clauses restrictives, les ventes d'actions d'entreprises fermées par rapport au prix subséquent observé sur les actions des mêmes entreprises lors d'une émission initiale et les placements privés. Pratt (2009) présente une revue complète des diverses estimations. La fourchette des estimations des escomptes pour le manque de liquidité est extrêmement large : de 7,23\% (Bajaj, Denis, Ferris et Sarin, 2001) à 75 \% (Paglia et Harjoto, 2010). Au Canada, l'analyse d'environ 3000 placements privés dans de petites sociétés ouvertes montre un escompte de $11,76 \%$, mais un quart des placements commande une prime, contraire à la notion d'escompte pour le manque de liquidité (Carpentier, L'Her et Suret, 2011). L'effet du manque de liquidité sur le coût du capital existe, mais est très difficile à modéliser et à quantifier. Nous ne traitons donc pas en détail de cette dimension.

\subsection{L'incapacité des modèles à expliquer les taux observés}

Même si ses hypothèses ne sont pas parfaitement respectées, un modèle peut être utile si ses prévisions correspondent à la réalité. Ce n'est pas le cas du CAPM. Il semble impossible de réconcilier les taux estimés à l'aide des modèles classiques avec ceux observés en pratique dans le cas des placements privés ou sur les marchés des actions de très petite capitalisation, assimilables aux nouvelles entreprises.

\subsubsection{Les taux exigés}

Manigart et al. (2002) situent les taux demandés par les investisseurs en capital de risque de différents pays entre 36 et $45 \%$ pour les phases de démarrage. Pratt et Grabowski (2010, p. 614) évoquent des taux de 50 à $70 \%$ pour cette même catégorie d'investisseurs aux États-Unis. Les anges investisseurs demanderaient des taux de 30 à 58 \% (Degennaro, 2011). Les estimations de primes de risque de marché se situent généralement entre 4 et $5 \%$ (Damodaran, 2010). Le taux sans risque est, depuis plusieurs années, 
de l'ordre de 3 à $4 \%$. Les taux observés en pratique ne peuvent donc être conciliés avec ceux prévus par le CAPM que si le niveau de risque systématique attaché au capital entrepreneurial atteint des valeurs supérieures à 10 , ce qui semble peu plausible. En effet, les fonds de placement privés, l'une des composantes du capital entrepreneurial dont le risque peut effectivement être estimé, ont en moyenne des niveaux de risque systématique proches de l'unité (Jegadeesh, Kräussl et Pollet, 2009). Il est donc impossible d'expliquer le coût du capital demandé par les investisseurs en capital de risque par le CAPM, même en incluant les primes de taille de 3 ou $4 \%$ le plus souvent évoquées.

\subsubsection{Les taux réalisés par les investisseurs privés}

Les taux de rendement réalisés par les anges investisseurs seraient positifs en moyenne, mais leur distribution rend très difficile l'interprétation des rendements moyens: les anges obtiennent des rendements négatifs dans 50 à $60 \%$ des cas (Degennaro, 2011). Environ le tiers de leurs investissements se traduit par une perte totale. Dans la moitié des cas, l'investisseur ne récupère pas toute sa mise de fonds. La médiane des taux de rendement est donc nulle. Toutefois, $14,5 \%$ des placements permettent aux anges de quintupler leur mise de fonds, et, dans 5,3\% des cas, la valeur de sortie représente plus de 20 fois l'investissement initial. Les résultats canadiens sont proches de ceux rapportés aux États-Unis, mais diffèrent selon les catégories d'anges investisseurs (Riding, 2008).

Entre 1995 et 2005, le taux de rendement annuel moyen du capital de risque a été de $-3 \%$ au Canada. Il a été de $-2,8 \%$ pour les dix ans se terminant en décembre 2008, selon les données de l'Association canadienne du capital de risque et d'investissement. On ne peut donc raisonnablement pas utiliser le taux réalisé pour estimer les taux requis sur ce type de placement au Canada. Le rendement des fonds dans les autres pays et pour les autres types de placement est également faible, même si l'industrie du capital de risque revendique généralement des rendements relativement élevés aux États-Unis. L'association américaine indique des taux de rendement internes de 22,49\% au cours des vingt dernières années (Cambridge Associates LLC, 2009), mais les travaux universitaires rigoureux contredisent ces énoncés (Korteweg et Sorensen, 2010; Phalippou et Gottschalg, 2009).

\subsubsection{Les taux réalisés sur les marchés publics de petite capitalisation}

$\mathrm{Au}$ Canada, les normes d'inscription en bourse très permissives, les mécanismes permettant de contourner les exigences de l'émission initiale et la présence d'un marché public de capital de risque font que de très nombreuses 
entreprises publiques sont des PME ou même des entreprises en démarrage (Carpentier, L'Her et Suret, 2010). Cette situation a permis d'analyser des taux de rendement suivant les divers types d'opérations de financement par des entreprises définies comme des PME selon l'Union européenne. Les émissions initiales (Carpentier, Cumming et Suret, 2012), les entrées en bourse par prise de contrôle inversée (Carpentier et Suret, 2012), les émissions subséquentes (Carpentier,L'Her et Suret,2012) et les placements privés (Carpentier et al., 2011) procurent des rendements moyens anormalement négatifs. Pour modéliser ces rendements correctement, il faut tenir compte d'autres facteurs de risque tels que la taille, les possibilités de croissance et la propension aux investissements (Carpentier et al., 2011). Cette situation s'observe également dans le cas des entreprises faisant une émission initiale sur une bourse conventionnelle, étape ultime du financement entrepreneurial (Eckbo, Masulis et Norli, 2007).

\subsubsection{Les taux réalisés par les entrepreneurs}

La majeure partie du capital des PME provient des entrepreneurs. Plusieurs études ont tenté de mesurer les revenus et les rendements de ceux-ci. Leurs revenus paraissent inférieurs à ceux de non-entrepreneurs de même qualification (Parker, 2009, chap. 13). La rentabilité de l'investissement entrepreneurial serait également inférieure à celle des indices boursiers, même si la diversification des portefeuilles est faible et le risque élevé (Hamilton, 2000; Moskowitz et Vissing-Jorgensen, 2002). Les ménages ayant des participations dans les entreprises y investissent généralement $70 \%$ de leur avoir. Le taux de rendement est proche de celui de l'ensemble du marché boursier. Le niveau de risque est cependant fort élevé: $36 \%$ des entreprises survivent dix ans. Hall et Woodward (2010) analysent l'ensemble des entreprises américaines ayant reçu du capital de risque aux États-Unis entre 1987 et 2008. Le rendement des entrepreneurs ayant lancé l'idée et démarré le projet serait nul dans $75 \%$ des cas. Ce rendement est très faible en moyenne lorsque le risque propre à ces projets est correctement pris en compte. L'entrepreneuriat est d'ailleurs parfois présenté comme une activité sans but lucratif (Benz, 2009).

Ces résultats surprennent. Les propriétaires de PME supportent des risques élevés et détiennent un portefeuille déséquilibré, ce qui accroît leur niveau de risque. Ils se satisfont pourtant d'un rendement qu'ils obtiendraient d'un fonds de placement indiciel. Le rendement du capital entrepreneurial ne rémunère pas correctement le risque de tels placements. Toutefois, la faiblesse relative des revenus et des rendements serait en partie imputable à la dissimulation d'une partie des revenus. Les entrepreneurs ne divulgueraient 
que 50 à $70 \%$ de leurs revenus totaux (Parker, 2009, p. 365). Sarada (2010) estime à $32 \%$ la proportion de revenus non déclarés par les entrepreneurs aux États-Unis. Une partie du rendement n'est pas mesurée, lorsque des bénéfices non pécuniaires sont tirés des entreprises. Les entrepreneurs tireraient de leur activité des satisfactions dépassant les avantages tangibles (Benz, 2009). Il est également très difficile de mesurer correctement le rendement du capital entrepreneurial en raison de la fiscalité (Gurley-Calvez et Bruce, 2008; Henrekson et Sanandaji, 2009).

Même s'ils exigent des taux de rendement élevés lors de l'investissement, les apporteurs de capital entrepreneurial réalisent en moyenne des rendements anormalement négatifs. Les entrepreneurs réalisent eux aussi des rendements anormalement faibles. Ce double constat constitue le paradoxe des capitaux privés (the private equity puzzle) évoqué par plusieurs auteurs. Ce paradoxe peut se définir ainsi: bien que présentant un risque élevé, le capital-actions investi dans les sociétés fermées procure de faibles rendements. Le CAPM n'étant pas utilisable pour estimer le coût du capital entrepreneurial, il faut utiliser des approches différentes. Une revue complète des travaux disponibles fait émerger quatre approches, traitées successivement. La méthode de l'empilage des primes, approche intuitive employée par les praticiens, et les méthodes basées sur l'estimation empirique des diverses primes reposent sur une idée commune: ajouter au modèle classique du CAPM diverses primes de risque. Inversement, la méthode du risque total et les approches probabilistes remettent en question la mesure du risque pertinente dans l'univers entrepreneurial.

\section{L'empilage de primes}

Cette approche, connue sous le terme de build-up method, est couramment utilisée par les évaluateurs professionnels et fréquemment acceptée par les tribunaux aux États-Unis.

\subsection{Cadre conceptuel}

Le cadre conceptuel est inexistant. Cette technique ne repose sur aucun modèle théorique. L'intuition qui la justifie est que le coût du capital découle de trois facteurs de risque, liés respectivement au marché, à la taille et à l'entreprise. Une variante de ce modèle introduit une prime sectorielle, capturant une partie du risque lié à l'entreprise. 


\subsection{Utilisation}

Diverses primes s'ajoutent au taux des obligations gouvernementales. Le modèle classique est le suivant (Hitchner, 2003, p. 87; Pratt et Grabowski, 2010; Trevino, 1997):

Taux sans risque

+ prime de risque de marché

+ prime de taille

+ prime de risque spécifique à l'entreprise

= coût du capital

Le rapport annuel de Grabowski (2010) est la source fréquente des estimations des diverses primes pour les praticiens. La prime de risque de marché est généralement de 4 à $5 \%$. Ce modèle ne tient pas compte du risque systématique, ce qui peut sembler logique dans un cadre n'incorporant pas de modèle de la relation rendement-risque. Le risque spécifique à l'entreprise est pris en compte par l'ajout d'une prime arbitraire.

La prime de taille est d'environ 3 à $4 \%$, sur la base des travaux menés dans l'univers des entreprises inscrites sur les bourses majeures aux ÉtatsUnis. C'est l'écart moyen séparant le rendement des plus petites et des plus grandes capitalisations américaines.

La détermination de la prime de risque spécifique à l'entreprise ne fait pas consensus, et il n'existe ni base théorique ni moyen d'en mesurer l'amplitude. Calvert et Smith (2011) analysent l'ensemble des travaux empiriques menés dans ce domaine et concluent qu'il n'existe aucune preuve attestant que cette prime de risque spécifique est rémunérée par le marché, ni dans le cas des sociétés ouvertes ni dans celui des sociétés fermées. Les praticiens utilisent toutefois fréquemment ce modèle (Pratt et Grabowski, 2010, chap. 7). Pour estimer la prime, ils emploient généralement une liste de facteurs de risque spécifique, auxquels sont attachées des valeurs numériques: le manque d'historique, la volatilité des bénéfices, la dépendance par rapport à un ou des employés clés ou le manque de profondeur de l'équipe de direction sont vus comme des facteurs augmentant ce risque, alors que l'existence de contrats à long terme le réduit (Hitchner 2003, p. 148). Calvert et Smith (2011, p. 18) associent cette approche par les facteurs de risque au folklore des praticiens.

\subsection{Critique des limites}

La méthode de l'empilage des primes souffre de plusieurs problèmes graves qui en limitent fortement la crédibilité. Premièrement, elle est très arbitraire. La prime spécifique, au centre de la méthode, ne repose sur aucun modèle et 
dépend totalement des opinions de l'utilisateur. La prime de taille est estimée au moyen de données de sociétés ouvertes du marché américain provenant des principales bases de données comptables et financières. L'application de ces primes aux situations des sociétés fermées est discutable, d'autant plus, comme le soulignent Pratt et Grabowski (2010, p. 95), que la prime de taille est estimée sur un univers n'incluant pas les petites entreprises. Les plus petites entreprises utilisées pour évaluer cette prime rapportent une capitalisation boursière d'environ 200 millions de dollars. Elles ont donc peu en commun avec les PME. Troisièmement, la méthode de l'empilage amène à compter plusieurs fois l'effet de certains facteurs: une petite entreprise peut se voir ainsi créditer d'une prime de taille et de risque spécifique, alors que les deux éléments sont liés. Enfin, il n'existe aucun moyen de lier les primes aux caractéristiques des entreprises, et la même prime de taille sera appliquée à l'ensemble des entreprises.

Pour estimer la prime de risque spécifique, des consultants américains ont présenté le Butler Pinkerton Calculator ${ }^{\mathrm{TM}}$ comme un instrument révolutionnaire pour les petites sociétés fermées (Butler et Pinkerton, 2006; Butler, 2009). Il s'agit d'un chiffrier reposant sur l'utilisation du risque total pour estimer le risque spécifique d'une entreprise. L'idée est d'extraire ce risque d'une équation du type : bêta total $=$ bêta classique + prime de taille + prime de risque spécifique. Cette méthode a soulevé un vif débat (Kasper, 2009; Wisehart, 2010). Sans entrer dans le détail, utiliser le risque total pour évaluer le coût du capital de sociétés fermées n'est pas une nouveauté. Cette méthode figure dans nombre de manuels et travaux (section 5).

L'intuition de l'ajout d'une prime de risque spécifique reste cependant intéressante. En effet, les PME constituent un ensemble hétérogène et leurs probabilités de succès et d'échec varient fortement. Les éléments retenus pour établir le niveau de risque spécifique (Butler, 2009, p. 3) paraissent raisonnables et proches de ceux employés dans les modèles de prédiction d'échec et de succès. Les modèles à facteurs constituent une approche plus rigoureuse que l'empilage des primes et reposent sur la même idée.

\section{Les modèles à facteurs appliqués au capital entrepreneurial}

\subsection{Intuition}

Le CAPM n'est pas parfaitement spécifié en dehors de l'univers des grandes entreprises composant les indices. Il est mal adapté pour estimer le coût du capital des petites sociétés fermées à forte croissance et expliquer les rende- 
ments postérieurs aux émissions d'actions (Eckbo et al., 2007, chap. 6). Les modèles à facteurs sont devenus la norme pour expliquer ces rendements. Diverses primes s'ajoutent à la prime du marché du CAPM.

\subsection{Cadre conceptuel}

Fama et French (1996) proposent le Three Factors Pricing Model (TFPM), extension du CAPM, reposant sur des bases purement empiriques et non sur une dérivation théorique (Estrada, 2011). Le TFPM explique le rendement des titres en tenant compte de deux «anomalies de marché» reconnues depuis plusieurs années: l'effet de taille (size effect ou small firm effect) et l'effet «book-to-market». Les titres de petite capitalisation ont, en moyenne, un rendement supérieur à celui des titres de grande capitalisation. Il y aurait donc une prime de taille. Les titres de valeur ont, en moyenne, un rendement supérieur à celui des titres dits de croissance. Les premiers ont un ratio de la valeur comptable des capitaux propres (book equity) à la valeur marchande (market equity) élevé, alors que ce ratio (book-to-market ou BM) est faible pour les seconds. Les banques constituent des titres de valeur, les jeunes entreprises technologiques, des titres de croissance.

Le TFPM s'écrit comme suit:

$\mathrm{E}\left(\mathrm{R}_{i}\right)-\mathrm{r}_{f}=\left(\mathrm{b}_{i}\left[\mathrm{E}(\mathrm{R} m)-\mathrm{r}_{f}\right]\right)+\left(\mathrm{s}_{i} * \mathrm{SMB}\right)+\left(\mathrm{h}_{i} * \mathrm{HML}\right)$

Avec:

$\left[\mathrm{E}(\mathrm{R} m)-\mathrm{r}_{f}\right]:$ Prime de marché

SMB: Prime de taille, soit l'écart de rendement entre les petites et les grandes entreprises (Small Minus Big).

HML: Prime liée au ratio BM, soit l'écart de rendement entre les entreprises à haut et à bas ratio BM (High Minus Low).

$\mathrm{b}_{i}, \mathrm{~s}_{i}$ et $\mathrm{h}_{i}$ : quantités (facteurs) de risque associées aux diverses primes pour l'entreprise $i$.

\subsection{Utilisation}

Utiliser ce modèle est lourd et réservé aux travaux empiriques, d'autant plus qu'un facteur d'initiative est généralement ajouté aux facteurs taille et croissance. On estime les primes totales, SMB et HML, pour l'ensemble du marché, en effectuant des tris successifs pour isoler les effets de chacun 
des deux facteurs. On évalue les facteurs $\mathrm{b}_{i}, \mathrm{~s}_{i}$ et $\mathrm{h}_{i}$ en estimant le modèle (équation 4) sur les périodes les plus longues possible. On vérifie ensuite si, en moyenne, les coefficients $\mathrm{b}_{i}, \mathrm{~s}_{i}$ et $\mathrm{h}_{i}$ sont statistiquement significatifs.

L'utilisation pratique du modèle ressemble à celle du CAPM: pour estimer le taux requis pour un titre $j$, on estime les primes grâce aux données passées, puis on calcule les coefficients $\mathrm{b}_{i}, \mathrm{~s}_{i}$ et $\mathrm{h}_{i}$. Ceux-ci indiquent la sensibilité du titre étudié au facteur correspondant. Il reste à prédire les primes futures et à leur appliquer les coefficients correspondants pour obtenir une estimation du coût du capital. Compte tenu de l'importance des calculs et du manque de données dans le cas des sociétés fermées, l'application pratique de ce modèle reste limitée. Utiliser les primes sans employer toutes les subtilités du modèle est cependant possible.

\subsection{Illustration}

Utiliser des primes de taille pour ajuster le coût du capital obtenu par le CAPM pose deux difficultés. Premièrement, ces primes sont systématiquement estimées à l'aide de données d'entreprises inscrites aux principales bourses. Deuxièmement, on oublie généralement de tenir compte des interactions entre les effets de croissance, de liquidité et de taille. Ainsi, la prime associée au ratio $\mathrm{BM}$ a un effet opposé à celui de la taille, mais il est le plus souvent négligé. Nous n'abordons ici que la première de ces difficultés.

Le tableau 1 synthétise les études proposant une estimation de la prime de taille. Selon Damodaran (2010, p. 34), à partir des données de French, entre 1927 et 2009, la prime de taille est économiquement significative. Le rendement des titres de petite capitalisation est supérieur à l'indice par $4,66 \%$ en moyenne. Inversement, l'écart entre le rendement des grandes firmes et l'indice est de $-4,08 \%$. Toutefois, la volatilité est énorme et le niveau de signification statistique est relativement faible. Les primes existent en moyenne (et sont utilisables ex ante), mais il ne faut pas s'attendre à les observer chaque année. Le minimum indique, par exemple, que le rendement des plus petits titres a été inférieur à celui de l'indice par $28,42 \%$ une année donnée. Grabowski (2010) rapporte des primes d'environ $11 \%$ pour les plus petites entreprises américaines en les divisant en 25 groupes.

Toutefois, ces petites entreprises américaines cotées ne sont pas des PME. La limite supérieure du décile des plus petites entreprises selon French est de 287 millions de dollars américains en 2010. L'actif moyen des plus petites entreprises analysées par Grabowski (2010) est de 115 millions de dollars américains environ. Les primes estimées sur de telles bases ne permettent pas d'estimer le coût du capital entrepreneurial, qui commande 
vraisemblablement des primes de taille plus élevées. Très peu d'études des primes incorporent les très petites entreprises. À notre connaissance, les seules estimations disponibles sont celles de Beedles (1992), qui mesure une prime de $6,06 \%$ par mois (72,72\% par année) pour les plus petites firmes australiennes, dont la capitalisation moyenne est de 1,5 million de dollars américains. Cette étude, menée entre 1974 et 1987, requiert cependant une mise à jour.

\section{TABLEAU 1}

Synthèse des études estimant la prime de taille

\begin{tabular}{llc}
\hline Étude & Échantillon & $\begin{array}{l}\text { Prime de taille } \\
\text { annuelle moyenne }\end{array}$ \\
\hline Damodaran (2010, p. 34) & États-Unis, 1927-2009 & $4,66 \%$ \\
Grabowski (2010) & États-Unis, 1963-2009 & $11,00 \%$ \\
Beedles (1992) & Australie, 1974-1987 & $72,72 \%$ \\
Carpentier et al. (2011) & Canada, 1993-2005 & $6,12 \%$ \\
\hline
\end{tabular}

La situation particulière canadienne, où de très nombreuses entreprises cotées sont des PME, permet d'estimer des primes de taille utilisables pour évaluer le coût du capital entrepreneurial. Fin 2006, la moitié des entreprises de la Bourse de croissance TSX avaient une valeur boursière inférieure à 9 millions de dollars (Carpentier et Suret, 2010).

Nous utilisons les rendements des entreprises canadiennes cotées disponibles dans Datastream entre 1986 et 2006. La valeur des actions est souvent très faible, et le taux de disparition des entreprises, fort élevé. Utiliser de tels rendements demande donc un travail méticuleux de vérification. Nous avons ainsi vérifié les changements de noms, de statut, les rendements supérieurs à $500 \%$ et éliminé les titres inactifs.

Nous avons trié les entreprises en fonction de leur capitalisation boursière en décembre de chaque année et constitué 11 groupes de taille (tableau 2). Pour chaque titre, nous avons ensuite calculé le rendement annuel composé de janvier à décembre, lorsque cinq rendements mensuels au moins étaient disponibles pour une année. La prime de taille annuelle est la différence entre le rendement moyen de chaque groupe de taille et celui du groupe 10, représentant le rendement du marché boursier. 
TABLEAU 2

Primes de taille entre janvier 1986 et décembre 2006 au Canada

\begin{tabular}{ccrrrrr}
\hline $\begin{array}{c}\text { Groupe } \\
\text { de taille d'entreprises }\end{array}$ & $\begin{array}{c}\text { Nombre } \\
\text { moyenne }\end{array}$ & $\begin{array}{c}\text { Capitalisation } \\
\text { médiane }\end{array}$ & $\begin{array}{c}\text { Capitalisation } \\
\text { moyen }\end{array}$ & $\begin{array}{c}\text { Rendement } \\
\text { écart type }\end{array}$ & $\begin{array}{c}\text { Prime } \\
\text { de taille }\end{array}$ \\
\hline 0 & 4306 & 0,33 & 0,24 & $88,30 \%$ & $249,14 \%$ & $75,17 \%$ \\
1 & 4520 & 0,81 & 0,62 & $55,77 \%$ & $228,28 \%$ & $42,64 \%$ \\
2 & 4616 & 1,45 & 1,10 & $41,44 \%$ & $183,28 \%$ & $28,31 \%$ \\
3 & 4695 & 2,46 & 1,79 & $34,09 \%$ & $183,97 \%$ & $20,96 \%$ \\
4 & 4735 & 4,04 & 2,88 & $22,80 \%$ & $163,07 \%$ & $9,67 \%$ \\
5 & 4726 & 6,89 & 4,83 & $17,14 \%$ & $130,86 \%$ & $4,01 \%$ \\
6 & 4758 & 12,27 & 8,85 & $15,23 \%$ & $129,44 \%$ & $2,10 \%$ \\
7 & 4769 & 23,94 & 17,66 & $13,85 \%$ & $112,07 \%$ & $0,72 \%$ \\
8 & 4794 & 52,92 & 41,27 & $14,30 \%$ & $93,44 \%$ & $1,17 \%$ \\
9 & 4811 & 149,71 & 123,37 & $10,99 \%$ & $64,27 \%$ & $-2,14 \%$ \\
10 & 4856 & 2324,25 & 767,12 & $13,13 \%$ & $46,90 \%$ & \\
\hline Total & 51586 & 234,42 & 5,51 & $29,01 \%$ & $156,37 \%$ & \\
\hline
\end{tabular}

Note: La capitalisation est exprimée en million de dollars.

Les rendements mensuels sont composés annuellement sur chaque année civile.

Nous estimons une prime de taille, applicable aux entreprises de très petite capitalisation, à environ $75 \%$ par année. Ces entreprises ont une capitalisation médiane de 240000 \$ canadiens. Les entreprises dont la valeur marchande des capitaux propres est entre un et trois millions de dollars (les cibles du capital de risque) justifient des primes de taille de 21 à $42 \%$. Le coût du capital se situerait donc entre 30 et $50 \%$, ce qui correspond bien au taux requis par les investisseurs en capital de risque.

\subsection{Limites et critiques}

Les estimations du tableau 1 représentent un avantage important par rapport aux estimations classiques basées sur les entreprises des principales bourses. Les valeurs rapportées sont fort différentes de celles le plus souvent employées. Au Canada, la prime de taille estimée sur les principaux marchés, entre 1993 et 2005, est de 6,12 \% (Carpentier et al., 2011). Toutefois, la seule prise en compte de la prime de taille n'est pas suffisante. 
Au moins deux autres primes devraient être ajoutées pour estimer le coût du capital entrepreneurial: les primes de croissance et de manque de liquidité. Les modèles empiriques indiquent que le facteur croissance joue un rôle important dans l'explication des rendements. Les entreprises dont le ratio $\mathrm{BM}$ est faible (titres de croissance) ont un rendement moyen inférieur à celui des titres de valeur. La prime associée à cette caractéristique est généralement du même ordre que la prime de taille, mais elle joue en sens inverse. Les entreprises en démarrage, notamment dans le secteur technologique, ont souvent de faibles valeurs comptables puisque les dépenses de R-D ne sont pas capitalisées. Elles sont des titres de croissance et commandent donc un taux de rendement moindre que celui de l'ensemble du marché. Lorsque la prime de taille est estimée et utilisée seule, cet effet conduit à surévaluer le coût du capital des petites entreprises. Les effets du manque de liquidité devraient également être pris en considération. Liu (2006) propose un CAPM augmenté des effets de taille, croissance et liquidité, et mesure une prime pour le manque de liquidité du même ordre que la prime de taille. Idéalement, les primes de taille, de croissance et de liquidité devraient être estimées de façon à en isoler les effets. Toutefois, l'estimation des effets croissance pour les petites entreprises pose d'importants problèmes, puisqu'il faut rapprocher les données comptables des données de marché de chacune des entreprises au cours de longues périodes. L'évaluation de la liquidité est également délicate, surtout lorsque les fréquences de transactions sont très faibles. C'est la raison pour laquelle nous nous sommes limités à ne présenter ici que les estimations de prime de taille.

Les travaux décrits ci-dessus permettent de défendre l'ajout d'une prime de taille à l'estimation du coût du capital. Toutefois, à l'exception des estimations présentées ici, les primes de taille disponibles dans les diverses études ne sont pas applicables au capital entrepreneurial. Lorsque l'approche par la prime de taille est utilisée, on considère implicitement que les entreprises d'une classe de taille donnée représentent des niveaux de risque équivalents. Or les probabilités d'échec auxquelles font face les investisseurs sont très différentes. Au lieu d'estimer des primes pour ajuster le coût du capital obtenu par le CAPM, le risque peut être mesuré autrement que par le bêta.

\section{L'approche du risque total}

\subsection{Intuition}

Le risque rémunéré, dans le cas des grandes entreprises, est le risque systématique puisque les investisseurs détiennent des portefeuilles diversifiés ou peuvent le faire à un coût minime. Ils ne sont donc pas rémunérés par le 
marché lorsqu'ils s'exposent au risque non systématique. Dans l'approche du risque total, le risque non systématique est rémunéré, car l'entrepreneur ne peut s'y soustraire.

\subsection{Cadre conceptuel}

L'approche par le risque total repose sur le constat de l'absence de diversification des portefeuilles des entrepreneurs. Ceux-ci ne sont généralement pas en mesure d'allouer une partie importante de leur richesse à la détention d'actif autre que les actions de leur entreprise. Puisque le dirigeant n'est généralement pas en mesure de détenir un portefeuille bien diversifié, le risque systématique n'est pas une mesure adéquate de risque du capital entrepreneurial. Il faut utiliser le risque total, mesurant la volatilité des rendements ou des flux monétaires. L'effet de diversification, permettant d'éliminer le risque non systématique, ne joue pas dans cette situation.

\subsection{Utilisation}

Damodaran (2002, p. 668) et Smith et al. (2011, p. 437) proposent d'utiliser le risque total, qu'ils nomment bêta total, pour estimer le risque puis le coût du capital d'une société fermée. Le risque total est mesuré par la volatilité relative des rendements, que l'on note $\sigma_{\mathrm{i}} / \sigma_{\mathrm{m}}$. Il exprime donc combien il y a d'unités de volatilité de marché dans la volatilité du titre.

On obtient le bêta total de deux façons équivalentes:

Bêta total $=$ bêta $«$ classique $» / \rho_{\text {im }}$

Bêta total $=\sigma_{\mathrm{i}} / \sigma_{\mathrm{m}}$

Avec:

Bêta «classique» = covariance $\mathrm{r}_{\mathrm{i}} \mathrm{r}_{\mathrm{m}} /$ variance des rendements du marché,

$\rho_{\text {im }}$ : corrélation entre les séries de rendement du titre

et du marché $=$ covariance $\mathrm{r}_{\mathrm{i}} \mathrm{r}_{\mathrm{m}} / \sigma_{\mathrm{i}} \sigma_{\mathrm{m}}$,

$\sigma_{\mathrm{i}}$ et $\sigma_{\mathrm{m}}$ sont les écarts types des séries de rendement du titre $\left(\mathrm{r}_{\mathrm{i}}\right)$ et du marché $\left(\mathrm{r}_{\mathrm{m}}\right)$.

Le coût du capital estimé du point de vue de l'entrepreneur $\left(\mathrm{R}_{\mathrm{ie}}\right)$ devient donc:

$\mathrm{E}\left(\mathrm{R}_{\mathrm{ie}}\right)=\mathrm{r}_{\mathrm{f}}+\sigma_{\mathrm{i}} / \sigma_{\mathrm{m}}\left(\mathrm{E}\left(\mathrm{R}_{\mathrm{M}}\right)-\mathrm{r}_{\mathrm{f}}\right)$ 
Alors que, du point de vue de l'investisseur externe parfaitement diversifié, il demeure:

$E\left(R_{i}\right)=r_{f}+\beta_{i}\left(E\left(R_{M}\right)-r_{f}\right)$

Le rendement requis par un investisseur ou un dirigeant partiellement diversifié devient une moyenne pondérée du taux donné par l'équation 7 et du rendement attendu du marché. Les poids sont les proportions investies respectivement dans l'entreprise et dans le portefeuille diversifié. Le bêta total représente le risque total relatif, et non plus le risque systématique. Il sera donc plus élevé que le bêta classique.

\subsection{Illustration}

Le tableau 3 illustre l'estimation du bêta, du bêta total et du coût du capital à l'aide de données provenant de dix entreprises canadiennes de petite taille ${ }^{4}$. Les rendements des entreprises sont très divers, de $-23,24 \%$ à $56,58 \%$ annuellement, alors que le rendement du marché a été de 16,98\% en moyenne pendant la période étudiée. Cette situation illustre le risque élevé, qui atteint 45,5\%, soit neuf fois celui du marché. Les bêtas classiques sont généralement faibles, voire négatifs, notamment pour les entreprises à faibles rendements. Les trois cas de bêtas très élevés sont associés à des entreprises ayant connu des rendements extrêmes au cours de quelques mois. Les bêtas classiques sont donc de piètres indicateurs du risque de ces petites entreprises. Les bêtas totaux sont, en moyenne, de 5,41 contre 0,92 pour les bêtas classiques. Le coût du capital estimé pour chacune des entreprises est donc largement supérieur lorsque ce modèle est appliqué. Il se situe à 30,05\% contre 7,62\% avec le CAPM, en utilisant une prime de risque de $5 \%$ et un taux sans risque de $3 \%$.

Kerins, Smith et Smith (2004) appliquent le modèle à un ensemble d'entreprises technologiques pour estimer les coûts d'opportunité selon le niveau de diversification des entrepreneurs et investisseurs externes. Les données boursières sont relevées à la suite des émissions initiales de ces entreprises. Avec un taux sans risque de $4 \%$ et un rendement attendu du marché boursier de $10 \%$, le rendement requis par l'investisseur parfaitement diversifié serait de $11,37 \%$. Il est de $57,5 \%$ pour le dirigeant ayant investi tout son patrimoine

4. Huit entreprises sont inscrites à la Bourse de croissance TSX; deux y sont aussi inscrites, mais leurs ventes sont inférieures à cinq millions de dollars pendant la période. Nous étudions la période 1995-1999, durant laquelle il ne survient pas d'événement de marché particulier comme en 2001,2002 et 2008. 
dans l'entreprise et de 45,6\% pour l'entrepreneur ayant investi $35 \%$ de son avoir dans le projet (Kerins et al.,2004, p. 402). Les investisseurs en capital de risque sont considérés comme parfaitement diversifiés, ce qui n'est pas le cas (Zhang et al.,2011). Ces auteurs refont l'analyse de Kerins et al. et montrent que le risque total des entreprises ayant effectué une émission initiale sans rapporter de bénéfices diminue fortement à mesure que ces entreprises franchissent les principaux jalons du développement. Deux articles confirment l'importance du manque de diversification dans la gestion financière des PME.

TABLEAU 3

Illustration des estimations de risque systématique, de risque total et de coût du capital selon les approches du CAPM et du risque total, pour 10 entreprises de petite taille, 1995-1999

\begin{tabular}{|c|c|c|c|c|c|c|c|}
\hline & \multicolumn{3}{|c|}{ Rendement } & \multicolumn{2}{|c|}{ Risque } & \multicolumn{2}{|c|}{ Coût du capital } \\
\hline & $\begin{array}{l}\text { Annuel } \\
\text { moyen géo- } \\
\text { métrique } \\
(\%)\end{array}$ & $\begin{array}{c}\text { Mensuel } \\
\text { moyen } \\
(\%)\end{array}$ & $\begin{array}{l}\text { Écart } \\
\text { type } \\
(\%)\end{array}$ & Bêta & $\begin{array}{l}\text { Bêta } \\
\text { total }\end{array}$ & $\begin{array}{c}\text { Selon le } \\
\text { CAPM } \\
(\%)\end{array}$ & $\begin{array}{c}\text { Selon } \\
\text { le risque } \\
\text { total } \\
(\%)\end{array}$ \\
\hline TSX & 16,98 & 1,43 & 4,84 & & & & \\
\hline Circa Enterprises & 5,57 & 1,32 & 13,64 & 0,02 & 2,82 & 3,08 & 17,10 \\
\hline Polymer Solutions & $-16,51$ & $-0,22$ & 16,40 & 0,64 & 3,39 & 6,21 & 19,95 \\
\hline Promatek Ind. & 34,10 & 3,59 & 17,26 & 0,76 & 3,57 & 6,80 & 20,84 \\
\hline RPM Tech. & $-0,82$ & 0,40 & 9,83 & $-0,13$ & 2,03 & 2,34 & 13,16 \\
\hline TML Venture & $-23,24$ & 0,60 & 23,94 & $-0,36$ & 4,95 & 1,21 & 27,75 \\
\hline Pacific Safety Prod. & $-7,20$ & 0,60 & 15,92 & $-0,12$ & 3,29 & 2,39 & 19,46 \\
\hline Spectra & $-3,15$ & 5,96 & 44,33 & 2,82 & 9,16 & 17,12 & 48,82 \\
\hline WE XL Holding & $-19,51$ & 2,30 & 35,15 & $-0,24$ & 7,27 & 1,80 & 39,33 \\
\hline Intl. Datacasting & 56,58 & 9,38 & 45,50 & 2,83 & 9,41 & 17,17 & 50,03 \\
\hline Mansfield Minerals & 4,98 & 5,99 & 39,74 & 3,02 & 8,22 & 18,12 & 44,08 \\
\hline Moyennes & & & & 0,92 & 5,41 & 7,62 & 30,05 \\
\hline
\end{tabular}

Les entrepreneurs ayant investi une plus grande proportion de leur avoir dans leur entreprise utilisent davantage le financement bancaire que les autres (Mueller, 2008). Cette observation corrobore l'hypothèse d'une relation positive entre l'absence de diversification et le coût du capital. 
En s'endettant davantage, les entrepreneurs peu diversifiés chercheraient à réduire le coût moyen pondéré du capital. Par ailleurs, les entrepreneurs peu diversifiés obtiendraient, en moyenne, des rendements supérieurs (Mueller, 2010). Cette observation est également compatible avec les idées suivantes: l'absence de diversification justifie une prime, et les entrepreneurs dont toute la richesse se trouve dans l'entreprise y consacrent les efforts maximums.

\subsection{Critiques et limites}

Si elle évite d'estimer des primes, l'approche du risque total pose plusieurs problèmes importants. Premièrement, ce modèle produit des estimations très élevées du coût du capital du dirigeant. Ces estimations sont difficilement conciliables avec les faibles rendements du capital entrepreneurial, notamment dans le cas des entreprises recevant du capital de risque (Hall et Woodward, 2010). Deuxièmement, le coût du capital de l'investisseur externe au portefeuille bien diversifié serait largement inférieur à celui du dirigeant. En effet, les investisseurs privés externes peuvent éliminer totalement le risque non systématique en diversifiant leur portefeuille. On se trouve donc dans la situation inconfortable où un projet commande des coûts du capital différents selon le statut de l'investisseur. L'investisseur externe diversifié devrait donc attribuer à l'entreprise une valeur supérieure à celle demandée par le dirigeant, et le rapport serait de l'ordre de un à quatre sur la base des estimations évoquées par Kerins et al. (2004). Cette observation ne correspond pas à la situation observée lors des négociations entre entrepreneurs et apporteurs de capital (Goldenberg et Goldenberg, 2009). Selon cette approche, le taux requis par un investisseur en capital de risque est à peine supérieur à celui du marché, ce que contredisent tous les relevés des taux exigés par ce type d'investisseur. Enfin, les estimations reposent sur des données boursières de sociétés ouvertes. On devra calculer le bêta total en utilisant les rendements d'entreprises cotées du même secteur (ou d'indices sectoriels) dont certains facteurs de risque et de liquidité sont très différents de ceux des entreprises naissantes.

L'intuition de cette approche de l'estimation du coût du capital reste cependant valable: le risque systématique n'est pas la mesure adéquate du risque des PME, compte tenu de l'absence de diversification. La volatilité des rendements des sociétés ouvertes est probablement un meilleur estimateur de ce risque, mais elle demeure une mesure incomplète et surtout peu commode du risque entrepreneurial. 


\section{Probabilités et coût du capital}

\subsection{Intuition}

En finance classique, on ne tient pas compte du risque d'échec des entreprises pour deux raisons. Premièrement, les faillites des entreprises inscrites en bourse dans la majorité des marchés sont des événements rares. Deuxièmement, l'investisseur classique peut limiter les effets d'un tel risque au moyen de la diversification. La situation est bien différente dans le cas de la finance entrepreneuriale, où la possibilité d'échec est importante. Si, en finance classique, les investisseurs et les dirigeants partagent les mêmes anticipations de flux monétaires futurs, ce n'est pas le cas dans les PME à forte croissance.

Utiliser des probabilités d'échec et de succès permet de réconcilier le taux utilisé par les investisseurs en capital de risque et les résultats des modèles classiques. Un entrepreneur sollicite un apport de capital externe lors d'un démarrage. Faute de revenus ou de bénéfices pour baser son évaluation, il propose d'employer la méthode des «venture capitalists». En multipliant les revenus (ventes) prévus dans cinq ans $\left(\mathrm{R}_{5}\right)$ par le multiple des revenus $(\mathrm{M}$, le rapport entre la valeur des entreprises et leurs revenus), on obtient la valeur probable de l'entreprise à la fin de l'horizon d'investissement. Actualiser cette valeur terminale (au taux $k$ ) donne la valeur de l'entreprise $\left(\mathrm{V}_{0}\right)$ :

$\mathrm{V}_{0}=\left(\mathrm{R}_{5} \times \mathrm{M}\right) /(1+k)^{5}$

Si $\mathrm{R}_{5}$ est de 10 millions de dollars, $\mathrm{M}$ de 1,3 et $k$ de $20 \%$, l'équation 9 permet d'évaluer l'entreprise à 5,22 millions de dollars.

L'investisseur expérimenté en démarrage d'entreprises connaît les probabilités d'échec et de succès. Il attribue une probabilité de $20 \%$ au scénario décrit par le promoteur, de $30 \%$ à un scénario où les revenus seront réduits de moitié et de $50 \%$ à l'échec du projet. Compte tenu de ces probabilités, appliquer l'équation 9 à chaque scénario donne une valeur de 1,8 million de dollars $=(0,2 \times 5,22)+(0,3 \times 2,61)+(0,5 \times 0)$.

Pour le dirigeant, une valeur de 1,8 million de dollars pour un projet valant 13 millions de dollars dans cinq ans revient à actualiser cette valeur future au taux de $48 \%$ par année.

Cet exemple illustre comment le taux élevé requis par les investisseurs externes tient compte des probabilités d'échec des projets en émergence. Le promoteur ne considère généralement que l'hypothèse optimiste. Les investisseurs considèrent l'ensemble des perspectives 
futures et les intègrent dans leur estimation du coût du capital. Leach et Melicher (2011, p. 252) évoquent ce phénomène de suroptimisme (venture hubris). Les investisseurs externes préfèrent en tenir compte en ajustant le taux, plutôt que de demander aux entrepreneurs d'ajuster leurs prévisions pour y incorporer la probabilité d'échec ${ }^{5}$. Ruhnka et Young (1991, p. 124) illustrent la relation entre les probabilités d'échec et le taux requis par les sociétés de capital de risque. Quelques formalisations de cette idée ont été proposées, reposant sur l'emploi des probabilités d'échec ou de succès (Cheung, 1999; Moro, Lucas et Kodwani, 2010), l'omission des possibilités d'échec dans l'analyse des projets (Ruback, 2011) ou la notion d'options réelles (Goldenberg et Goldenberg, 2009). Cette avenue de recherche, peu développée, semble prometteuse. Les probabilités d'échec et de succès des PME sont relativement bien connues. Si l'on tient correctement compte du risque en établissant les scénarios, on peut actualiser les divers flux au taux sans risque. Nous n'exposerons ici que la méthode proposée par Cheung (1999) à partir d'un modèle développé pour estimer le taux requis sur les obligations de pacotille.

\subsection{Cadre conceptuel}

Cheung (1999) utilise un cadre de neutralité au risque, semblable à celui employé dans les modèles d'évaluation des options. Dans un tel cadre, les probabilités capturent la totalité du risque et on peut donc actualiser les flux au taux sans risque ${ }^{6}$. L'analyse porte sur plusieurs périodes, et la probabilité de survie de l'entreprise à chacune de ces périodes est au centre du modèle. Elle est notée $p$. Il s'agit de la probabilité pour qu'une entreprise donnée, ayant survécu $x$ années, disparaisse à la période $t+1$.

Pour simplifier, même si l'on sait que les probabilités d'échec diminuent avec les années, Cheung estime dans un premier temps que $p$ est constant. La plupart des travaux portant sur la survie des entreprises établissent une probabilité de survie après $n$ années; on passe facilement de cette probabilité cumulée à la probabilité annuelle, si l'on suppose que celle-ci est constante. Si la probabilité de défaut à 10 ans est $40 \%$, la probabilité de survie annuelle

5. Les scénarios et les probabilités sont toutefois utilisés pour évaluer les entreprises naissantes dans la méthode dite First Chicago Method (Steffens et Douglas, 2007).

6. Les hypothèses du modèle sont:1) les investisseurs et prêteurs sont neutres au risque ;2) si l'entreprise disparaît, les actionnaires ne reçoivent rien;3) les créanciers reçoivent un rendement inférieur au taux sans risque en cas de liquidation de l'entreprise; 4) il n'y a pas d'impôt. 
est donnée par $1-p^{10}=0,40$, donc $p^{10}=0,60$ et $p=0,95$. La probabilité qu'une entreprise fasse faillite au cours d'une année donnée est de l'ordre de $5 \%$, $\operatorname{car}(1-p)=0,05$.

Dans le cas simple d'une seule période, si $\mathrm{r}_{e}$ est le taux de rendement requis sur les capitaux propres et $r_{f}$ le taux sans risque, on peut montrer que: $\left(1+\mathrm{r}_{e}\right)=\left(1+r_{f}\right) / p$

Si le taux sans risque est de $5 \%$ et la probabilité de survie de $60 \%$, l'investisseur va exiger un taux de rendement de $75 \%$. En effet, $\mathrm{r}_{e}=\left[\left(1+\mathrm{r}_{f}\right) / p\right]$ $-1=[1,05 / 0,6]-1$. Si l'entreprise ne verse aucun dividende, alors cette formule est valable quel que soit le nombre de sous-périodes, dans la mesure où la probabilité est constante durant tout l'horizon d'analyse. Le taux requis n'est pas une fonction de l'horizon.

Si la probabilité de survie change, il faut définir $p_{1}$, valable pendant la période de 1 à $n$ et une probabilité $p_{2}$ ensuite. Dans un tel cas, le taux requis est:

$\mathrm{r}_{e}=\left(1+r_{f}\right) \times\left(p_{2}^{-1 / n}\right) \times\left(p_{1}^{(1-n) / n}\right)-1$

\subsection{Illustration}

Le modèle n'exige, en intrant, que l'estimation de la probabilité de survie de l'entreprise, comparée à celle d'entreprises similaires. Les résultats de l'application du modèle apparaissent au tableau 4 , lorsque la probabilité $p_{1}$ est valable pour les cinq premières années.

Les méthodes basées sur les probabilités d'échec et de succès des entreprises présentent l'avantage de reposer sur des ensembles d'information relativement complets. Au Québec, le ministère du Développement économique et de l'Innovation (MDEIE, 2008) rapporte des données sur la survie des nouvelles entreprises. Les taux canadiens sont régulièrement publiés par Statistique Canada (Baldwin, Bian, Dupuy et Gellatly, 2000). L'Organisation de coopération et de développement économiques (OCDE, 2006) fournit des données sur la naissance et la survie des entreprises. Les échecs et succès des émissions initiales sont également utilisables lorsque celles-ci sont effectuées par de petits émetteurs, comme au Canada (Carpentier et Suret, 2011; St-Pierre, 2000). Les caractéristiques des entrepreneurs sont liées aux probabilités de survie et d'échec (Santarelli et Vivarelli, 2007). Il semble donc possible de raffiner les statistiques globales. 
TABLEAU 4

Ajustement des taux requis par les investisseurs aux probabilités de survie de l'entreprise, avec $\mathbf{r}_{f}=5 \%$ et $n=5$, d'après Cheung (1999, p. 334)

\begin{tabular}{cccc}
\hline $\begin{array}{c}\text { Probabilité } \\
\text { annuelle de survie } \\
\left(\boldsymbol{p}_{\mathbf{1}}\right)\end{array}$ & $\begin{array}{c}\text { Probabilité } \\
\text { annuelle de survie } \\
\text { en période 2 }\left(\boldsymbol{p}_{\mathbf{2}}\right)\end{array}$ & $\begin{array}{c}\text { Taux requis, } \\
\text { probabilité } \\
\text { constante }\end{array}$ & $\begin{array}{c}\text { Taux requis, } \\
\text { avec deux périodes } \\
\text { distinctes }\end{array}$ \\
\hline $100,00 \%$ & $100,00 \%$ & $5,00 \%$ & $5,00 \%$ \\
$95,00 \%$ & $95,00 \%$ & $10,53 \%$ & $10,53 \%$ \\
$90,00 \%$ & $95,00 \%$ & $16,67 \%$ & $15,41 \%$ \\
$85,00 \%$ & $95,00 \%$ & $23,53 \%$ & $20,81 \%$ \\
$80,00 \%$ & $95,00 \%$ & $31,25 \%$ & $26,82 \%$ \\
$75,00 \%$ & $95,00 \%$ & $40,00 \%$ & $33,54 \%$ \\
$70,00 \%$ & $95,00 \%$ & $50,00 \%$ & $41,11 \%$ \\
$65,00 \%$ & $95,00 \%$ & $61,54 \%$ & $49,73 \%$ \\
$60,00 \%$ & $95,00 \%$ & $75,00 \%$ & $59,63 \%$ \\
\hline
\end{tabular}

Grabowski (2010, p. 40) fait une tentative en ce sens en classant les entreprises en fonction de leur score $\mathrm{Z}$ de Altman, puis en estimant les primes de risque. Les différences de rendement observées entre les groupes de score $\mathrm{Z}$ sont cependant faibles, ce qui indique vraisemblablement que le score, développé pour prédire les faillites, n'est pas un indicateur fiable du risque perçu par les investisseurs.

\subsection{Limites et critiques}

Le modèle est dérivé dans un cadre de neutralité au risque; cette limite n'est cependant pas majeure. Ainsi, on peut modifier le modèle pour que le taux de base ne soit pas $\mathrm{r}_{f}$, mais le taux produit par le CAPM, en éliminant ainsi cette restriction. La faiblesse du modèle nous semble être liée à la forme très simplifiée des états d'occurrence. Il existe toute une série d'états intermédiaires entre le succès et l'échec d'une entreprise, et l'évolution couvre plusieurs sous-périodes. Cette évolution peut être modélisée en temps continu, sur la base des outils développés pour évaluer les options réelles (Levasseur, 2005). Toutefois, le modèle devient très complexe et difficile à mettre en œuvre. On peut également utiliser un cadre discret multipériodique, comme le suggère Ruback (2011) dans le cas de l'évaluation de projets. Les modèles tenant compte des probabilités constituent une avenue prometteuse de recherche. 


\section{Conclusion}

Nous avons évoqué les quatre méthodes d'estimation du coût du capital entrepreneurial mentionnées dans les travaux scientifiques et dans les manuels destinés aux praticiens. Nous avons mis en évidence les relations existant entre ces approches. L'idée générale est unique: il faut mesurer le risque du capital entrepreneurial, puis associer une ou des primes à ce risque. Les approches divergent en matière d'indicateurs de risque et de primes. L'avenir de ces domaines importants de recherche passe vraisemblablement par une intégration des trois approches, qui présentent des intuitions intéressantes et des lacunes importantes. Pour le moment, aucune des avenues proposées ne semble satisfaisante, ce qui nous conduit à suggérer plusieurs pistes de recherche.

L'empilage des primes permet de tenir compte des caractéristiques propres à chaque entreprise et d'utiliser des facteurs de risques particuliers. Les facteurs de risque et leur effet sur le taux requis sont toutefois arbitraires. En outre, la prime de taille est généralement estimée de façon approximative. L'étude des facteurs de risque et de leur relation avec les succès et échecs futurs a déjà été abordée, mais nous semble nécessiter des travaux complémentaires qui incorporeraient la notion de taux requis ou de valeur des entreprises.

Nous avons présenté des données originales relatives aux primes de taille pour les très petites entreprises. De telles estimations permettraient de défendre des primes plus cohérentes avec l'univers des sociétés fermées que celles généralement utilisées. Toutefois, des travaux complémentaires sont nécessaires pour élaborer des primes qui tiennent compte des effets de croissance, vraisemblablement liés aux probabilités de faillite (Griffin et Lemmon, 2002; Vassalou et Yuhang Xing, 2004). Les effets conjugués du manque de liquidité et de la taille des entreprises sur les rendements devraient également être distingués.

Le modèle d'estimation du coût du capital basé sur les probabilités représente une avenue intéressante, très partiellement explorée. Elle rejoint la méthode de l'empilage en formalisant la relation entre le risque spécifique et le taux requis. Elle est également cohérente avec certains aspects des modèles à facteurs, lorsqu'on admet que le facteur de croissance de même que l'effet de la taille sont associés aux probabilités de faillite (Vassalou et Yuhang Xing, 2004). Cette avenue de recherche présente l'avantage de reposer sur des données concernant les entreprises fermées et d'éviter l'énorme problème que pose l'estimation du coût du capital entrepreneurial à partir de données de marché. 
Pour conserver à ce texte une taille raisonnable, nous avons choisi de ne pas traiter en profondeur certains sujets importants. C'est le cas des effets potentiels du manque de liquidité sur le coût du capital et des effets possibles du cadre institutionnel sur ce même coût. Les cadres réglementaire et institutionnel semblent influencer le coût du capital des grandes entreprises (Hail et Leuz, 2006) et les possibilités de financement des entreprises naissantes (Chavis, Klapper et Love, 2010). Il est donc probable que le coût du capital entrepreneurial soit sensible au cadre institutionnel. Les résultats obtenus en interrogeant les investisseurs en capital de risque semblent indiquer que ces différences existent (Manigart et al., 2002). Toutefois, les difficultés d'estimation du coût du capital entrepreneurial sont considérablement plus grandes que celles, déjà importantes, auxquelles on se heurte pour estimer le coût du capital des grandes entreprises. Lier le cadre institutionnel au coût du capital entrepreneurial représente donc une autre avenue de recherche qui devrait être explorée.

\section{Bibliographie}

ACHARAYA, V.V. et L.H. PEDERSEN (2005), «Asset pricing with liquidity risk», Journal of Financial Economics, vol. 77, n² 2, p. 375-410.

AMIHUd, Y., H. MENDELSON et L.H. PEDERSEN (2005), «Liquidity and asset prices », Foundations and Trends in Finance, vol. 1, no 4, p. 269-364.

ANGEl CAPital EduCATiOn Fundation (2010), «Important things for entrepreneurs to know about angel investors », Présentation, $<$ http://www. angelcapitalassociation.org/data/Documents/Press \%20Center/What \%20 Ents \%20Should \%20Know \%20About \%20Angels \%202009.pdf>.

BAJAJ, M., D.J. DENIS, S.P.FERRIS et A. SARIN (2001), «Firm value and marketability discounts », Journal of Corporation Law, n 27, p. 89-115.

BALDWIN, J.R., L. BIAN, R. DUPUY, et G. GELLATLY (2000), «Failure rates for new Canadian firms: new perspectives on entry and exit», Statistics Canada, $n^{\circ}$ 61-526-XIE.

BEEDLES, W.L. (1992), «Small firm equity cost: evidence from Australia», Journal of Small Business Management, vol. 30, $\mathrm{n}^{\circ}$ 3, p. 57-65.

BENZ, M. (2009), «Entrepreneurship as a non-profit-seeking activity», The International Entrepreneurship and Management Journal, vol. 5, n ${ }^{\circ}$ 1, p. 23-44.

BUTLER, P. et K. PINKERTON (2006), «Company-specific risk - A different paradigm: a new benchmark», Business Valuation Review, vol. 25, n ${ }^{\circ}$, p. 22-28.

Butler, P.J. (2009), «The Butler Pinkerton Calculator: the choice», ASA Advanced Business Valuation Conference, Boston, octobre.

CALVERT, B. et D.C. SMITH (2011), «Company-specific risk premiums: update on the scholarly evidence», SSRN Working Paper. 
CAMBRIDGE ASSOCIATES LLC (2009), «U.S. venture capital index and selected benchmark statistics (March 31)», Rapport NVCA.

CARPENTIER, C., D.J. CUMMING et J.-M. SURET (2012), «The value of capital market regulation and certification: IPOs versus reverse mergers », Journal of Empirical Legal Studies, vol. 9, $\mathrm{n}^{\circ} 1$.

CARPENTIER, C., J.-F. L'HER et J.-M. SURET (2010), «Stock exchange markets for new ventures », Journal of Business Venturing, vol. 25, n 4, p. 403-422.

CARPENTIER, C., J.-F. L'HER et J.-M. SURET (2011), «Private investment in small public equity», Small Business Economics, Forthcoming, DOI:10.1007/s11187011-9707-7.

CARPENTIER, C., J.-F. L'HER et J.-M. SURET (2012), «Seasoned equity offerings by small and medium sized enterprises », Small Business Economics, vol. 38, n 4 , p. $449-465$.

CARPENTIER, C. et J.-M. SURET, (2010), «The Canadian public venture capital market», Venture Capital: Investment Strategies, Structures, and Policies, Hoboken, N.J., Wiley, p. 501-524.

CARPENTIER, C. et J.-M. SURET (2011), «The survival and success of Canadian penny stock IPOs», Small Business Economics, vol. 36, nº 1, p. 101-121.

CARPENTIER, C. et J.-M. SURET (2012), «Entrepreneurial equity financing and securities regulation : an empirical analysis », International Small Business Journal, vol. 30, n $^{\mathrm{o}} 1$, p. 41-64.

CHAVIS, L.W., L.F. KLAPPER et I. LOVE (2010), «The impact of the business environment on young firm financing», World Bank Policy Research Working Paper, $\mathrm{n}^{\circ} 5322$.

CHEUnG, J. (1999), «A probability based approach to estimating costs of capital for small business », Small Business Economics, vol. 12, no 4, p. 331-637.

COMMISSION EUROPÉENNE (2003), «Entrepreneurship in Europe», Green Paper $<$ http://eur-lex.europa.eu/LexUriServ/LexUriServ.do?uri=COM:2003:0027: FIN:EN:PDF>

DAMODARAN, A. (2002), Investment Valuation, New York, Wiley.

DAMODARAN, A. (2008), «What is the riskfree rate? A search for the basic building block», SSRN Working paper.

DAMODARAN, A. (2010), «Equity risk premiums: determinants, estimation and implications - the 2010 edition », SSRN Working paper.

DEAVES, R., C. DINE et W. HORTON (2006), «How investment decisions are made?», Report commmissioned by the task force to modernize securities legislation in Canada, <http://www.tfmsl.ca/docs/V2(3)\%20Deaves.pdf $>$.

DEGENNARO, R.P. (2011), «Angel investors and their investments », SSRN Working paper.

DENIS, D.J. (2004), «Entrepreneurial finance: an overview of the issues and evidence», Journal of Corporate Finance, vol. 10, n 2, p. 301-326. 
DONG, M., J.-S. MICHEL et J.A. PANDES (2011), «Underwriter quality and long-run IPO performance », Financial Management, vol. 40, n 1, p. 219-251.

ECKBO, B., R. MASUlis et O. NORLI (2007), «Security offerings: a survey», Handbook of Corporate Finance: Empirical Corporate Finance, vol. 1, p. 233-373.

ESTRADA, J. (2011), «The three-factor model: a practitioner's guide», Journal of Applied Corporate Finance, vol. 23, n 2, p. 77-84.

FAMA, E.F. et K.R. FRENCH (1996), «Multifactor explanations of asset pricing anomalies », The Journal of Finance, vol. 51, n ${ }^{\circ}$ 1, p. 55-85.

FRANZIONI, F.A., E. NOWAK et L. PHALIPPOU (2011), «Private equity performance and liquidity risk», Swiss Finance Institute Research Paper, nº 09-43.

GOLDENBERG, D.H. et M.D. GOLDENBERG (2009), «Why entrepreneurs and VCs disagree in valuing start-up firms : imputing the target rate of return using DCF vs. option-based approaches », Journal of Private Equity, vol. 13, nº 1, p. 73-79.

GOMPERS, P. et J. LERNER (2010), «Equity financing», Handbook of Entrepreneurship Research, New York, Springer, p. 183-214.

GRABOWSKI, R. (2010), «Risk premium report», Report, Duff \& Phelps, LLC.

GRIFFIN, J.M. et M.L. LEMMON (2002), «Book to market equity, distress risk, and stock returns », The Journal of Finance, vol. 57, n 5, p. 2317-2336.

GURLEY-CALVEZ, T. et D. BRUCE (2008), «Do tax cuts promote entrepreneurial longevity?», National Tax Journal, vol. 61, n² 2, p. 225-250.

HAIL, L. et C. LEUZ (2006), «International differences in the cost of equity capital: do legal institutions and securities regulation matter?», Journal of Accounting Research, vol. $44 \mathrm{n}^{\circ} 3$, p. 485-531.

HALL, R.E. et S.E. WOODWARD (2010), «The burden of the nondiversifiable risk of entrepreneurship », American Economic Review, vol. 100, n 3, p. 1163-1194.

HAMilton, B.H. (2000), «Does entrepreneurship pay? An empirical analysis of the returns to self-employment », Journal of Political Economy, vol. 108, n 3 , p. 604-631.

HellwiG, M. (1980), «On the aggregation of information in competitive markets », Journal of Economic Theory, vol. 22, $\mathrm{n}^{\circ}$ 3, p. 477-498.

HENREKSON, M. et T. SANANDAJI (2009), «Entrepreneurship and the theory of taxation», Small Business Economics, vol. 37, n 2, p. 1-19.

HitCHNER, J.R. (2003), Financial Valuation, Hoboken, New Jersey, John Wiley \& Sons.

JEGADEESH, N., R. KRÄUSSL et J.M. POLLET (2009), « Risk and expected returns of private equity investments: evidence based on market prices », NBER Working Paper 15335.

KASPER, L. (2009), «Anomalous findings from the Butler Pinkerton model for company specific risk premiums », Monography, $28^{e}$ conférence annuelle ASA [vu sur Internet: Paper presented at the Advance Business Valuation Conference of the ASA], Boston, mai. 
KERINS, F., J.K. SMITH et R. SMITH (2004), «Opportunity cost of capital for venture capital investors and entrepreneurs», Journal of Financial and Quantitative Analysis, vol. 39, n 2, p. 385-405.

KORTEWEG, A. et M. SORENSEN (2010), «Risk and return characteristics of venture capital-backed entrepreneurial companies », Review of Financial Studies, vol.23, $\mathrm{n}^{\mathrm{o}} 10, \mathrm{p} .3738-3772$.

LEACH, J.C. et R.W. MELICHER (2011), Entrepreneurial Finance, $4^{\mathrm{e}}$ édition, Cincinnati, Ohio, South-Western Publishing.

LEVASSEUR, M. (2005), «Évaluation et coût du capital d'une start-up », La Revue du Financier, $\mathrm{n}^{\circ} 155$, septembre-octobre.

L'HER, J.-F. et J.-M. SURET (1991), «Hétérogénéité des anticipations, ventes à decouvert et prix des titres: une revue de la littérature », Finance, vol. 12, no 1 , p. $127-158$.

L'HER, J.-F. et J.-M. SURET (1995), «Heterogeneous expectations, short sales regulation, and the risk-return relationship », The Financial Review, vol. 30, no 4, p. 637-662.

LIU, W. (2006), «A liquidity-augmented capital asset pricing model», Journal of Financial Economics, vol. 82, n 3, p. 631-671.

LOCKE, S.M. et K. GUPTA (2008), «The performance of entrepreneurial companies post-listing on the New Zealand stock exchange», Venture Capital, vol. 10, $\mathrm{n}^{\circ} 1$, p. 87-110.

Manigart, S., K.D. Waele, M. Wright, K. Robbie, P. Desbrièeres, H. SAPIENZA et A. BEEKMAN (2002), «Determinants of required return in venture capital investments : a five-country study », Journal of Business Venturing, vol. 17, $\mathrm{n}^{\circ}$ 4, p. 291-312.

MiLLER, E.M. (1977), «Risk, uncertainty, and divergence of opinions », The Journal of Finance, vol. 32, $\mathrm{n}^{\mathrm{o}}$ 4, p. 1151-1168.

MINISTÈRE DU DÉVELOPPEMENT ÉCONOMIQUE, DE L'INNOVATION ET DE L'EXPORTATION (2008), «Taux de survie des nouvelles entreprises au Québec», Rapport, MDEIE Québec.

MORO, A., M. LUCAS et D. KODWANI (2010), «Small and medium sized enterprises (SMEs) and their cost of capital», International Conference on Management of SMEs 2010, Nagpur, Inde.

MOSKOWITZ, T.J. et A.VISSING-JORGENSEN (2002), «The returns to entrepreneurial investment: a private equity premium puzzle ? », American Economic Review, vol. $92, \mathrm{n}^{\circ}$ 4, p. 745-778.

MuEller, E. (2008), «How does owners' exposure to idiosyncratic risk influence the capital structure of private companies? », Journal of Empirical Finance, vol. $15, \mathrm{n}^{\circ}$ 2, p. 185-198.

MUELlER, E. (2010), «Returns to private equity - idiosyncratic risk does matter!», Review of Finance, vol. 15, n 3 , p. 1-30. 
ORGANISATION DE COOPÉRATION ET DE DÉVELOPPEMENT ÉCONOMIQUES (2006), Statistiques structurelles et démographiques des entreprises 2006, Paris, Éditions OCDE.

PAGLIA, J.K. et M. HARJOTO (2010), «The discount for lack of marketability in privately owned companies: a multiples approach», Journal of Business Valuation and Economic Loss Analysis, vol. 5, $\mathrm{n}^{\mathrm{o}} 1$.

PARKER, S.C. (2009), The Economics of Entrepreneurship, Cambridge, Cambridge University Press.

PhAlipPOU, L. et O. GOTTSCHALG (2009), «The performance of private equity funds », Review of Financial Studies, vol. 4, n² 22, p. 1747-1776.

PRATT, S.P. (2009), Business Valuation Discounts and Premiums, $2^{\mathrm{e}}$ édition, Hoboken, New Jersey, John Wiley \& Sons.

PRATT, S.P. et R.J. GRABOWSKI (2010), Cost of Capital: Applications and Examples, Hoboken, New Jersey, John Wiley \& Sons.

REDIS, J. et J.-L.PARÉ (2009), «L'émergence du champ de la finance entrepreneuriale: état des lieux et perpectives », $\mathrm{VI}^{\mathrm{e}}$ Congrès de l'Académie de l'Entrepreneuriat, 19, 20 et 21 novembre 2009, Sophia Antipolis.

RIDING, A.L. (2008), «Business angels and love money investors: segments of the informal market for risk capital», Venture Capital: An International Journal of Entrepreneurial Finance, vol. 10, nº 4, p. 355-369.

RUBACK, R.S. (2011), «Downsides and DCF: valuing biased cash flow forecasts », Journal of Applied Corporate Finance, vol. 23, n 2, p. 8-17.

RUHNKA, J.C. et J.E. YOUNG (1991), «Some hypotheses about risk in venture capital investing», Journal of Business Venturing, vol. 6, n 2, p. 115-133.

SANTARELLI, E. et M. VIVARELLI (2007), «Entrepreneurship and the process of firms' entry, survival and growth», Industrial and Corporate Change, vol. 16, n 3 , p. 455-488.

SARADA, F. (2010), «The unobserved returns to entrepreneurship», UC San Diego: Department of Economics, UCSD, Working Paper.

SÉJOURNÉ, B. (2006), «Pourquoi le comportement des épargnants français est-il si peu conforme à la théorie traditionnelle du portefeuille?», Autorité des marchés financiers (France), Les Cahiers scientifiques, $\mathrm{n}^{\circ} 1$.

SHANE, S.A. (2003), A General Theory of Entrepreneurship, Northampton, Mass., E. Elgar.

SMITH, J.K., R.L. SMITH et R.T. BLISS (2011), Entrepreneurial Finance: Strategy, Valuation, and Deal Structure, Stanford, Cal., Stanford University Press.

STEFFENS, P.R. et E.J. DOUGLAS (2007), «Valuing technology investments : use real options thinking but forget real options valuation», International Journal of Technoentrepreneurship, vol. 1, $\mathrm{n}^{\mathrm{o}}$ 1, p. 58-77.

ST-PIERRE, J. (2000), «Can the stock market success of Montreal's IPOs be predicted by the prospectus' content?», Small Business Economics, vol. 15, n 1, p.13-26. 
TREVINO, G.A. (1997), «Note on formulating and corroborating discount rates for small firms », Journal of Legal Economics, vol. 7, n 3, p. 45-54.

VASSALOU, M. et M. YUHANG XING (2004), «Default risk in equity returns», The Journal of Finance, vol. 59, n 2, p. 831-868.

WiseheART, D.P. (2010), «Boston's battle of the beta», Financial Valuation and Litigation Expert, vol. 22, p.12-15.

YAZDIPOUR, R. (2011), «A behavioral finance approach to decision making in entrepreneurial finance», The Journal of Entrepreneurial Finance, vol.13, $\mathrm{n}^{\circ} 2$, p. 56-75.

ZHANG, S., Q. HUO, D. SUN, D. WEI et S. XU (2011), «Profit milestones and changing risks and expected returns of venture capital projects: an empirical exploration using comparable companies », SSRN Working paper. 